\title{
Recent progress in hadronic light-by-light scattering
}

\author{
Martin Hoferichter ${ }^{1, *}$ \\ ${ }^{1}$ Albert Einstein Center for Fundamental Physics, Institute for Theoretical Physics, University of Bern, \\ Sidlerstrasse 5, 3012 Bern, Switzerland
}

\begin{abstract}
In recent years, significant progress in the calculation of the HLbL contribution to the anomalous magnetic moment of the muon has been achieved both with data-driven methods and in lattice QCD. In these proceedings I will discuss current developments aimed at controlling HLbL scattering at the level of $10 \%$, as required for the final precision of the Fermilab E989 experiment.
\end{abstract}

\section{Introduction}

The $4.2 \sigma$ tension between the experimental world average [1-5]

$$
a_{\mu}^{\exp }=116592061(41) \times 10^{-11}
$$

and the Standard-Model prediction for the anomalous magnetic moment of the muon [6-33]

$$
a_{\mu}^{\mathrm{SM}}=116591810(43) \times 10^{-11}
$$

continues to motivate further improvements in controlling the hadronic contributions, both hadronic vacuum polarization (HVP) and hadronic light-by-light scattering (HLbL). While for the HVP contribution a key challenge concerns reconciling the data-driven determination as reviewed in Ref. [6] and the lattice-QCD calculation by BMWc [34], see Refs. [35-39], for HLbL scattering the phenomenological determination, based on Refs. [19-31, 40-45], agrees well with the lattice calculation by RBC/UKQCD [32] already included in Ref. [6] and the more recent calculation by the Mainz group [46]. Here we discuss mainly the prospects and challenges involved in improving the data-driven determination further towards $10 \%$ precision, as required for the final goal of the Fermilab E989 experiment [47] and beyond [48, 49].

\section{White paper status}

The current status of the phenomenological determination as described in the White Paper (WP) [6] is given in Table 1 in comparison with previous compilations [42, 50-52]. The final recommended value was obtained by combining errors for the well-controlled lowenergy contributions-pseudoscalar poles [23, 26-28] and leading two-pion intermediate states $[24,25]$ - in quadrature, to the effect that the subleading contributions-scalar, tensor, and axial-vector states as well as short-distance contributions [19, 29-31, 40-45]—dominate

\footnotetext{
*e-mail: hoferichter@itp.unibe.ch
} 


\begin{tabular}{crrrr}
\hline Contribution & $\mathrm{PdRV}(09)$ & $\mathrm{N} / \mathrm{JN}(09)$ & $\mathrm{J}(17)$ & $\mathrm{WP} 20$ \\
\hline$\pi^{0}, \eta, \eta^{\prime}$-poles & $114(13)$ & $99(16)$ & $95.45(12.40)$ & $93.8(4.0)$ \\
$\pi, K$-loops/boxes & $-19(19)$ & $-19(13)$ & $-20(5)$ & $-16.4(2)$ \\
$S$-wave $\pi \pi$ rescattering & $-7(7)$ & $-7(2)$ & $-5.98(1.20)$ & $-8(1)$ \\
\hline subtotal & $88(24)$ & $73(21)$ & $69.5(13.4)$ & $69.4(4.1)$ \\
\hline scalars & - & - & - & $-1(3)$ \\
tensors & - & - & $1.1(1)$ & $6(6)$ \\
axial vectors & $15(10)$ & $22(5)$ & $7.55(2.71)$ & $15(10)$ \\
$u, d, s$-loops/short-distance & - & $21(3)$ & $20(4)$ & $3(1)$ \\
\hline$c$-loop & 2.3 & - & $2.3(2)$ & $92(19)$ \\
\hline total & $105(26)$ & $116(39)$ & $100.4(28.2)$ &
\end{tabular}

Table 1. Estimates for the various contributions to HLbL scattering from WP20 [6], PdRV(09) [50], N/JN(09) [51, 52], J(17) [42]; from Ref. [6].

the error budget. In particular, the uncertainties for these contributions were added linearly, to account for the fact that, contrary to the low-energy part, the bookkeeping is not yet based on a dispersive reasoning [20-22,53], which introduces ambiguities and thus sizable correlations among the errors. The total number $a_{\mu}^{\mathrm{HLbL}}$ (phenomenology) $=92(19) \times 10^{-11}$ agrees well with lattice QCD (for $q=u, d, s)-a_{\mu}^{\mathrm{HLbL}}(\mathrm{RBC} / \mathrm{UKQCD})=79(35) \times 10^{-11}$ [32] and $a_{\mu}^{\mathrm{HLbL}}(\mathrm{Mainz})=106.8(14.7) \times 10^{-11}$ [46]. The combination of phenomenology and $\mathrm{RBC} / \mathrm{UKQCD}$ (accounting for the role of the $c$-loop) then leads to the final recommendation $a_{\mu}^{\mathrm{HLbL}}(\mathrm{WP})=90(17) \times 10^{-11}[6]$.

\section{Pseudoscalar poles}

For the $\pi^{0}$ pole evaluations from dispersion relations [26, 27], Canterbury approximants [23], and lattice QCD [28] are available

$$
\begin{aligned}
a_{\mu}^{\pi^{0} \text {-pole }} & \left.\right|_{\text {dispersive }}=63.0_{-2.1}^{+2.7} \times 10^{-11}, & \left.a_{\mu}^{\pi^{0} \text {-pole }}\right|_{\text {Canterbury }}=63.6(2.7) \times 10^{-11}, \\
\left.a_{\mu}^{\pi^{0} \text {-pole }}\right|_{\text {lattice }+ \text { PrimEx }} & =62.3(2.3) \times 10^{-11}, & \left.a_{\mu}^{\pi^{0} \text {-pole }}\right|_{\text {lattice }}=59.7(3.6) \times 10^{-11},
\end{aligned}
$$

which agree at a level well below the required precision goal. The agreement is further improved if the experimental normalization for $\pi^{0} \rightarrow \gamma \gamma$ from the PrimEx experiment [54] is imposed in the lattice calculation. In addition, the singly-virtual limit of the pion transition form factor (TFF) agrees with the measurement from the BESIII experiment [55].

The situation differs for $\eta, \eta^{\prime}$, for which the WP number currently derives from Canterbury approximants alone [23], calling for further corroboration from dispersion relations and lattice QCD, both of which are in progress. On the lattice side, calculations for the $\eta, \eta^{\prime}$ are noisier than for the $\pi^{0}$, but are being addressed by (at least) two collaborations [56, 57]. On the dispersive side, the main challenge concerns so-called factorization breaking contributions, which can arise from a left-hand-cut structure involving the $a_{2}$ resonance. Such contributions cannot be addressed in the Canterbury approach, and thus it becomes critical to clarify their impact. A first step in this direction, relying on data for $e^{+} e^{-} \rightarrow \eta \pi \pi$, was recently performed in Ref. [58], with the main result that more differential data are required to resolve these contributions conclusively. 

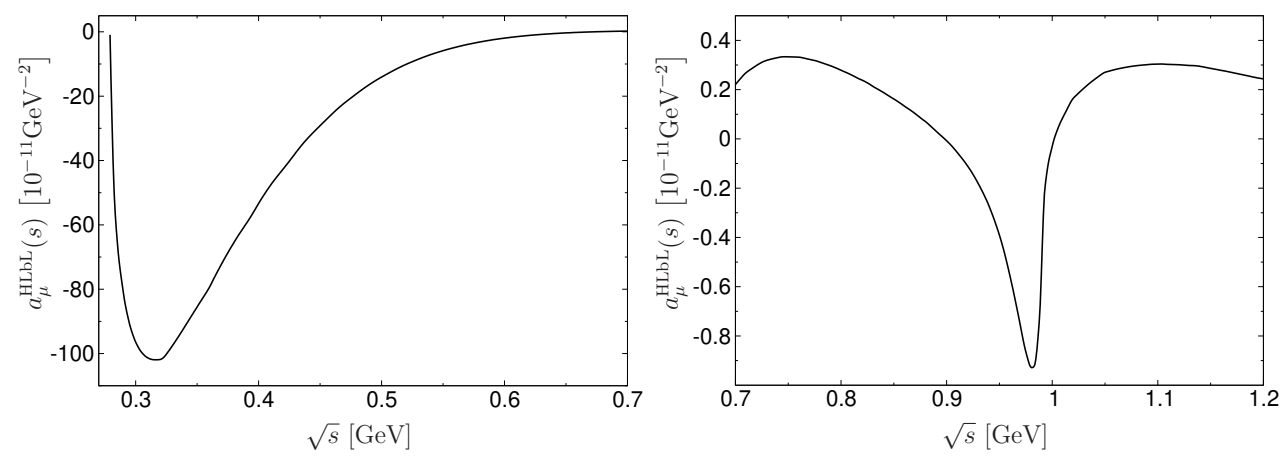

Figure 1. Spectral shape in the $f_{0}(500)$ (left) and the $f_{0}(980)$ (right) regions, as a function of center-ofmass energy $\sqrt{s}$; from Ref. [59]. The integration over $s$ gives the contribution to $a_{\mu}^{\mathrm{HLbL}}$.

\section{Scalar contributions and $\pi \pi$ rescattering}

In general, the contribution from single-particle poles to HLbL scattering depends on the choice of the basis for the HLbL tensor [25], with only the entire HLbL tensor basis independent by virtue of sum rules that may receive contributions from several (narrow) resonances of different quantum numbers at a time. Only pseudoscalar poles constitute an exception, making scalar resonances the first non-trivial test case. In Refs. [24, 25] it was shown that the dominant such contribution- $S$-wave $\pi \pi$ rescattering, which for isospin $I=0$ implements the $f_{0}(500)$ resonance in a model-independent way-is largely basis independent by itself, since the two helicity components cancel in the corresponding sum rule to a large extent. Recently, this approach was generalized to the partial-wave helicity amplitudes for $\gamma^{*} \gamma^{*} \rightarrow \pi \pi / \bar{K} K$ [60-65], which allows one to also describe the $f_{0}(980)$ and compare to a narrow-width approximation (NWA) [59]:

$$
\left.a_{\mu}^{\mathrm{HLbL}}\left[f_{0}(980)\right]\right|_{\text {rescattering }}=-0.2(1) \times 10^{-11},\left.\quad a_{\mu}^{\mathrm{HLbL}}\left[f_{0}(980)\right]\right|_{\mathrm{NWA}}=-0.37(6) \times 10^{-11},
$$

where the TFFs from the quark model of Ref. [66] have been used, in line with the expected asymptotic behavior [67]. This shows that the result from the NWA comes out reasonably close to the full dispersive implementation, suggesting that at least for sufficiently narrow states a similar approach should be viable as well for axial-vector and tensor resonances. In addition, it was found that the combined contribution from $S$-waves, including also the $a_{0}(980)$, amounts to $a_{\mu}^{\mathrm{HLbL}}$ [scalars] $=-9(1) \times 10^{-11}$ [59], with the effect of yet heavier states small and very uncertain due to the lack of reliable TFF input. Given that heavy scalar contributions are not expected to play a special role in the implementation of short-distance constraints (SDCs), contrary to axial-vector resonances, and first enter $\gtrsim 1.5 \mathrm{GeV}$, such effects are best considered part of the asymptotic matching [29, 68, 69].

\section{Axial-vector contributions and short-distance constraints}

SDCs are available in two kinematic regimes, (i) one in which all three non-vanishing virtualities are large, and (ii) another in which one is much smaller than the other two. The result for the former case has only recently been put onto solid footing by demonstrating that the perturbative QCD quark loop does arise as the first term in a well-defined operator product expansion (OPE) [29]. While non-perturbative corrections prove negligible [68], the 


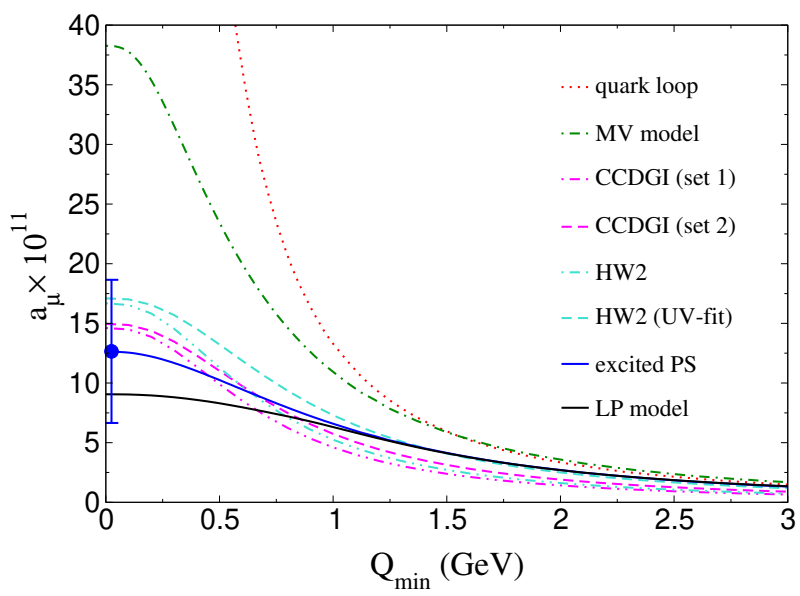

Figure 2. Comparison of the longitudinal short-distance contributions as a function of a common lower cutoff $Q_{\min }$ on the virtualities in the HLbL loop integral. The red dashed line refers to the perturbative QCD quark loop, the others to Ref. [19] (MV), Ref. [71] (CCDGI), Ref. [70] (HW2), Refs. [30, 31] (excited PS), and Ref. [74] (LP); from Ref. [75].

perturbative $\alpha_{s}$ corrections provide valuable insights into the onset of the asymptotic regime, typically scaling approximately as $1-\alpha_{s} / \pi$ [69].

The SDCs in regime (ii) were derived in Ref. [19], with subsequent discussions how the respective constraints should be implemented. The simple model proposed in Ref. [19] remains valid in the chiral limit, but its simplicity comes at the price of neglecting $2 \pi$ and $3 \pi$ singularities that strongly affect the low-energy region of the HLbL integral. This was pointed out in Refs. [30, 31], in which a Regge model based on excited pseudoscalar mesons was proposed, exploiting the fact that these are well controlled theoretically and can provide a viable implementation at physical quark masses. Alternatively, a model for a tower of resonances in holographic QCD has been proposed [70,71], which allows one to identify the model from Ref. [19] in a particular limit, while demonstrating at the same time that an additional contribution is necessary to fulfill the Landau-Yang theorem [72, 73], thus avoiding a sizable effect in the low-energy region. Third, an approach based on interpolants has been proposed in Ref. [74]. There is broad agreement among all three approaches as concerns the impact of the longitudinal SDCs, leading to contributions significantly smaller than the model from Ref. [19] would predict. A critical comparison of the different approaches can be found in Ref. [75], leading to the situation illustrated in Fig. 2.

In contrast, the role of the transverse SDCs, closely related to axial-vector degrees of freedom, is less well understood. There are a number of papers that analyze axial-vector contributions in a Lagrangian model $[40,42,45,76]$, but the combination with contributions evaluated in a dispersive framework remains to be understood. In particular, while it is possible to find a HLbL basis in which the axial-vector contributions coincide with the Lagrangian model [75], due to sum rules this choice of basis affects other contributions as well, and these consequences need to be carefully investigated.

In addition, information on the axial-vector TFFs is scarce, with the main source of information from the L3 measurements [77, 78] of $e^{+} e^{-} \rightarrow e^{+} e^{-} A, A=f_{1}, f_{1}^{\prime}$, with additional input from $f_{1} \rightarrow \rho \gamma[79,80], f_{1} \rightarrow \phi \gamma$ [80,81], and, most recently, $f_{1} \rightarrow e^{+} e^{-}$[82]. In particular, an improved measurement of the latter process would be extremely valuable to 

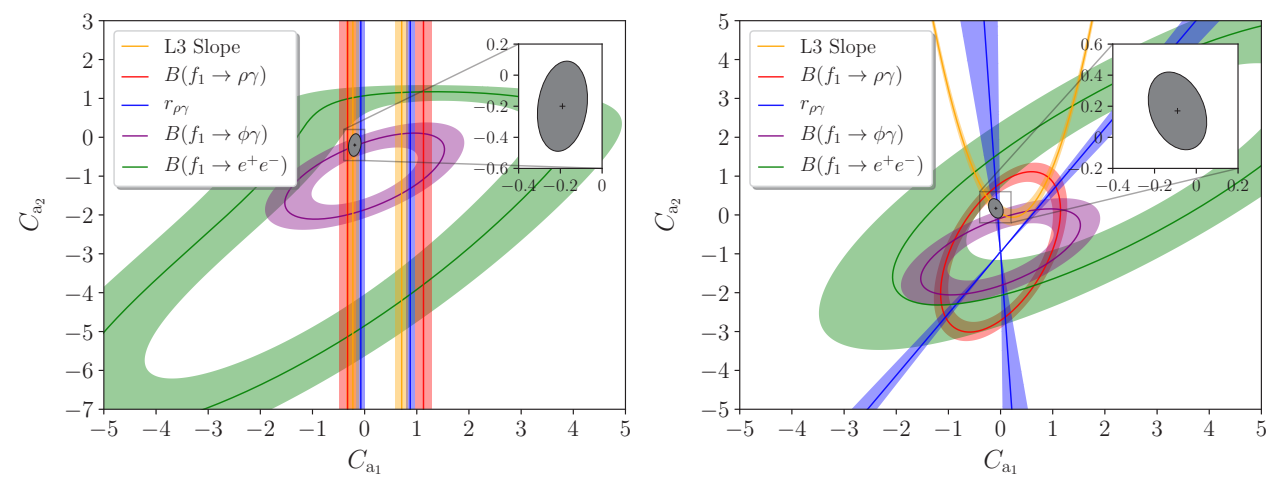

Figure 3. Constraints on the antisymmetric TFFs of the $f_{1}(1285)$ in terms of couplings $C_{\mathrm{a}_{1}}, C_{\mathrm{a}_{2}}$, for two variants of vector-meson-dominance parameterizations; from Ref. [83].

disentangle different TFFs, given that $e^{+} e^{-} \rightarrow e^{+} e^{-} A$ is primarily sensitive to the symmetric TFF only. A global analysis for the $f_{1}(1285)$, for which the experimental situation is best, was recently presented in Ref. [83], see Fig. 3. Estimates for the $f_{1}(1420)$ and the $a_{1}(1260)$ can then be obtained from $U(3)$ symmetry, with the mixing angle determined by the L3 measurements of the equivalent two-photon decay widths.

\section{Conclusions}

In these proceedings I reviewed the current status of HLbL scattering and discussed recent developments that aim at improving the precision towards the $10 \%$ level. While controlling subleading contributions in a data-driven way becomes increasingly challenging, recent work shows that it should be possible to achieve this goal with a combination of rigorous shortdistance constraints and experimental input on the subleading intermediate states. Anticipating similar progress in lattice QCD, there should be two independent methods available to determine the HLbL contribution at the required level, with detailed comparisons potentially allowing for further improvements in precision.

I am grateful to Gilberto Colangelo, Bastian Kubis, and Peter Stoffer for longstanding collaboration on the subjects reviewed here and for carefully reading this manuscript. Support by the Swiss National Science Foundation, under Project No. PCEFP2_181117, is gratefully acknowledged.

\section{References}

[1] G.W. Bennett et al. (Muon $g$ - 2), Phys. Rev. D 73, 072003 (2006), hep-ex/0602035

[2] B. Abi et al. (Muon $g-2$ ), Phys. Rev. Lett. 126, 141801 (2021), 2104.03281

[3] T. Albahri et al. (Muon $g$ - 2), Phys. Rev. D 103, 072002 (2021), 2104 . 03247

[4] T. Albahri et al. (Muon $g$ - 2), Phys. Rev. A 103, 042208 (2021), 2104.03201

[5] T. Albahri et al. (Muon g-2), Phys. Rev. Accel. Beams 24, 044002 (2021), 2104.03240

[6] T. Aoyama et al., Phys. Rept. 887, 1 (2020), 2006.04822

[7] T. Aoyama, M. Hayakawa, T. Kinoshita, M. Nio, Phys. Rev. Lett. 109, 111808 (2012), 1205.5370

[8] T. Aoyama, T. Kinoshita, M. Nio, Atoms 7, 28 (2019) 
[9] A. Czarnecki, W.J. Marciano, A. Vainshtein, Phys. Rev. D 67, 073006 (2003), [Erratum: Phys. Rev. D 73, 119901 (2006)], hep-ph/0212229

[10] C. Gnendiger, D. Stöckinger, H. Stöckinger-Kim, Phys. Rev. D 88, 053005 (2013), 1306.5546

[11] M. Davier, A. Hoecker, B. Malaescu, Z. Zhang, Eur. Phys. J. C 77, 827 (2017), 1706.09436

[12] A. Keshavarzi, D. Nomura, T. Teubner, Phys. Rev. D 97, 114025 (2018), 1802.02995

[13] G. Colangelo, M. Hoferichter, P. Stoffer, JHEP 02, 006 (2019), 1810.00007

[14] M. Hoferichter, B.L. Hoid, B. Kubis, JHEP 08, 137 (2019), 1907.01556

[15] M. Davier, A. Hoecker, B. Malaescu, Z. Zhang, Eur. Phys. J. C 80, 241 (2020), [Erratum: Eur. Phys. J. C 80, 410 (2020)], 1908.00921

[16] A. Keshavarzi, D. Nomura, T. Teubner, Phys. Rev. D 101, 014029 (2020), 1911.00367

[17] B.L. Hoid, M. Hoferichter, B. Kubis, Eur. Phys. J. C 80, 988 (2020), 2007.12696

[18] A. Kurz, T. Liu, P. Marquard, M. Steinhauser, Phys. Lett. B 734, 144 (2014), 1403.6400

[19] K. Melnikov, A. Vainshtein, Phys. Rev. D 70, 113006 (2004), hep-ph/0312226

[20] G. Colangelo, M. Hoferichter, M. Procura, P. Stoffer, JHEP 09, 091 (2014), 1402 . 7081

[21] G. Colangelo, M. Hoferichter, B. Kubis, M. Procura, P. Stoffer, Phys. Lett. B 738, 6 (2014), 1408.2517

[22] G. Colangelo, M. Hoferichter, M. Procura, P. Stoffer, JHEP 09, 074 (2015), 1506.01386

[23] P. Masjuan, P. Sánchez-Puertas, Phys. Rev. D 95, 054026 (2017), 1701.05829

[24] G. Colangelo, M. Hoferichter, M. Procura, P. Stoffer, Phys. Rev. Lett. 118, 232001 (2017), 1701.06554

[25] G. Colangelo, M. Hoferichter, M. Procura, P. Stoffer, JHEP 04, 161 (2017), 1702.07347

[26] M. Hoferichter, B.L. Hoid, B. Kubis, S. Leupold, S.P. Schneider, Phys. Rev. Lett. 121, 112002 (2018), 1805.01471

[27] M. Hoferichter, B.L. Hoid, B. Kubis, S. Leupold, S.P. Schneider, JHEP 10, 141 (2018), 1808.04823

[28] A. Gérardin, H.B. Meyer, A. Nyffeler, Phys. Rev. D 100, 034520 (2019), 1903.09471

[29] J. Bijnens, N. Hermansson-Truedsson, A. Rodríguez-Sánchez, Phys. Lett. B 798, 134994 (2019), 1908.03331

[30] G. Colangelo, F. Hagelstein, M. Hoferichter, L. Laub, P. Stoffer, Phys. Rev. D 101, 051501 (2020), 1910.11881

[31] G. Colangelo, F. Hagelstein, M. Hoferichter, L. Laub, P. Stoffer, JHEP 03, 101 (2020), 1910.13432

[32] T. Blum, N. Christ, M. Hayakawa, T. Izubuchi, L. Jin, C. Jung, C. Lehner, Phys. Rev. Lett. 124, 132002 (2020), 1911 . 08123

[33] G. Colangelo, M. Hoferichter, A. Nyffeler, M. Passera, P. Stoffer, Phys. Lett. B 735, 90 (2014), 1403.7512

[34] S. Borsanyi et al., Nature 593, 51 (2021), 2002 . 12347

[35] C. Lehner, A.S. Meyer, Phys. Rev. D 101, 074515 (2020), 2003.04177

[36] A. Crivellin, M. Hoferichter, C.A. Manzari, M. Montull, Phys. Rev. Lett. 125, 091801 (2020), 2003.04886

[37] A. Keshavarzi, W.J. Marciano, M. Passera, A. Sirlin, Phys. Rev. D 102, 033002 (2020), 2006.12666 
[38] B. Malaescu, M. Schott, Eur. Phys. J. C 81, 46 (2021), 2008.08107

[39] G. Colangelo, M. Hoferichter, P. Stoffer, Phys. Lett. B 814, 136073 (2021), 2010.07943

[40] V. Pauk, M. Vanderhaeghen, Eur. Phys. J. C 74, 3008 (2014), 1401.0832

[41] I. Danilkin, M. Vanderhaeghen, Phys. Rev. D 95, 014019 (2017), 1611.04646

[42] F. Jegerlehner, Springer Tracts Mod. Phys. 274, 1 (2017)

[43] M. Knecht, S. Narison, A. Rabemananjara, D. Rabetiarivony, Phys. Lett. B 787, 111 (2018), 1808.03848

[44] G. Eichmann, C.S. Fischer, R. Williams, Phys. Rev. D 101, 054015 (2020), 1910.06795

[45] P. Roig, P. Sánchez-Puertas, Phys. Rev. D 101, 074019 (2020), 1910.02881

[46] E.H. Chao, R.J. Hudspith, A. Gérardin, J.R. Green, H.B. Meyer, K. Ottnad, Eur. Phys. J. C 81, 651 (2021), 2104 . 02632

[47] J. Grange et al. (Muon $g-2$ ) (2015), 1501.06858

[48] M. Abe et al., PTEP 2019, 053C02 (2019), 1901.03047

[49] M. Aiba et al. (2021), 2111.05788

[50] J. Prades, E. de Rafael, A. Vainshtein, Adv. Ser. Direct. High Energy Phys. 20, 303 (2009), 0901.0306

[51] A. Nyffeler, Phys. Rev. D 79, 073012 (2009), 0901.1172

[52] F. Jegerlehner, A. Nyffeler, Phys. Rept. 477, 1 (2009), 0902 . 3360

[53] M. Hoferichter, G. Colangelo, M. Procura, P. Stoffer, Int. J. Mod. Phys. Conf. Ser. 35, 1460400 (2014), 1309.6877

[54] I. Larin et al. (PrimEx-II), Science 368, 506 (2020)

[55] M. Ablikim et al. (BESIII), Chin. Phys. C 44, 040001 (2020), 1912.05983

[56] S. Burri (2021), https://indico.cern.ch/event/1006302/contributions/ 4373340/attachments/2287903/3888993/burri_lattice21.pdf

[57] W. Verplanke (2021), https://indico.cern.ch/event/1006302/ contributions/4375437/attachments/2288167/3889500/VERPLANKE_ LATTICE2021.pdf

[58] S. Holz, J. Plenter, C.W. Xiao, T. Dato, C. Hanhart, B. Kubis, U.G. Meißner, A. Wirzba, Eur. Phys. J. C 81, 1002 (2021), 1509. 02194

[59] I. Danilkin, M. Hoferichter, P. Stoffer, Phys. Lett. B 820, 136502 (2021), 2105.01666

[60] R. García-Martín, B. Moussallam, Eur. Phys. J. C 70, 155 (2010), 1006.5373

[61] M. Hoferichter, D.R. Phillips, C. Schat, Eur. Phys. J. C 71, 1743 (2011), 1106.4147

[62] B. Moussallam, Eur. Phys. J. C 73, 2539 (2013), 1305.3143

[63] I. Danilkin, M. Vanderhaeghen, Phys. Lett. B 789, 366 (2019), 1810.03669

[64] M. Hoferichter, P. Stoffer, JHEP 07, 073 (2019), 1905 . 13198

[65] I. Danilkin, O. Deineka, M. Vanderhaeghen, Phys. Rev. D 101, 054008 (2020), 1909.04158

[66] G.A. Schuler, F.A. Berends, R. van Gulik, Nucl. Phys. B 523, 423 (1998), hep-ph/9710462

[67] M. Hoferichter, P. Stoffer, JHEP 05, 159 (2020), 2004 . 06127

[68] J. Bijnens, N. Hermansson-Truedsson, L. Laub, A. Rodríguez-Sánchez, JHEP 10, 203 (2020), 2008. 13487

[69] J. Bijnens, N. Hermansson-Truedsson, L. Laub, A. Rodríguez-Sánchez, JHEP 04, 240 (2021), 2101.09169

[70] J. Leutgeb, A. Rebhan, Phys. Rev. D 101, 114015 (2020), 1912.01596 
[71] L. Cappiello, O. Catà, G. D’Ambrosio, D. Greynat, A. Iyer, Phys. Rev. D 102, 016009 (2020), 1912.02779

[72] L.D. Landau, Dokl. Akad. Nauk SSSR 60, 207 (1948)

[73] C.N. Yang, Phys. Rev. 77, 242 (1950)

[74] J. Lüdtke, M. Procura, Eur. Phys. J. C 80, 1108 (2020), 2006.00007

[75] G. Colangelo, F. Hagelstein, M. Hoferichter, L. Laub, P. Stoffer, Eur. Phys. J. C 81, 702 (2021), 2106. 13222

[76] P. Masjuan, P. Roig, P. Sánchez-Puertas (2020), 2005 . 11761

[77] P. Achard et al. (L3), JHEP 03, 018 (2007)

[78] P. Achard et al. (L3), Phys. Lett. B 526, 269 (2002), hep-ex/0110073

[79] D.V. Amelin et al., Z. Phys. C 66, 71 (1995)

[80] P.A. Zyla et al. (Particle Data Group), PTEP 2020, 083C01 (2020)

[81] S.I. Bityukov et al., Phys. Lett. B 203, 327 (1988)

[82] M.N. Achasov et al. (SND), Phys. Lett. B 800, 135074 (2020), 1906.03838

[83] M. Zanke, M. Hoferichter, B. Kubis, JHEP 07, 106 (2021), 2103.09829 